\title{
Triple-negative breast cancer: New potential therapeutics derived from SOCS3 protein
}

\author{
Sara La Manna, Daniela Marasco, Giancarlo Morelli \\ University of Naples Federico II, Italy
}

https://doi.org/10.17952/35EPS.2018.270

Suppressor of cytokine signaling (SOCS) proteins are a family of negative feedback regulators of the JAK-STAT pathway [1]. SOCS1,2,3 and CIS are the members of this family but, only SOCS1 and SOCS3, contain a kinase inhibitory region (KIR) essential for the inactivation of JAKs, bringing to the suppression of inflammatory cytokines [2]. SOCS proteins seem to play an important role in inflammatory diseases and in development and progression of cancers. Particularly, neonatal fatal inflammatory disease is associated with SOCS1 protein deficiency [3] while its over-expression in experimental autoimmune encephalitis (EAE) reduces negative effects of IFN- $\gamma[4]$; instead, the recombinant SOCS3 revealed able to inhibit triple negative breast cancer (TNBC) tumour growth and metastasis by suppressing inflammatory cytokines signaling while the proteolytic degradation of SOCS3 protein in TNBC subtype leads to the activation of inflammatory cytokines [5]. In last years, many studies have been carried out on the KIR region of SOCS1 providing both mimetic and antagonist compounds [6] [7].

Concerning SOCS3, structural and functional investigations showed that it simultaneously binds JAK2 and the cytokine receptor to which it is attached indicating the specificity of SOCS3 action and explaining why SOCS3 inhibits only a subset of cytokines. Recently, with the intention to develop new potential targets of JAK-STAT pathway, thanks to the X-ray structure of the protein complex among JAK2 kinase domain, mouse SOCS3 and gp130 phoshotyrosine peptide [8], a series of SOCS3'mimetic peptides derived from the Nterminal region of SOCS3, covering two domain, KIR and extended SH2 subdomain (ESS), located at the interface of the complex with JAK2, have been designed [9].

These peptides have been in vitro characterized by Circular Dichroism (CD) and Surface Plasmon Resonance (SPR) spectroscopies. In particular, the polypeptide formed by the union of KIR and ESS fragments, named KIRESS, showed in Figure 1, demonstrated to be enhanced in the helical content with respect to shorter protein fragments, KIR and ESS peptides. At the same time, in direct binding assays it revealed to have the highest affinity toward JAK2, showing a micromolar $\mathrm{K}_{\mathrm{D}}$.
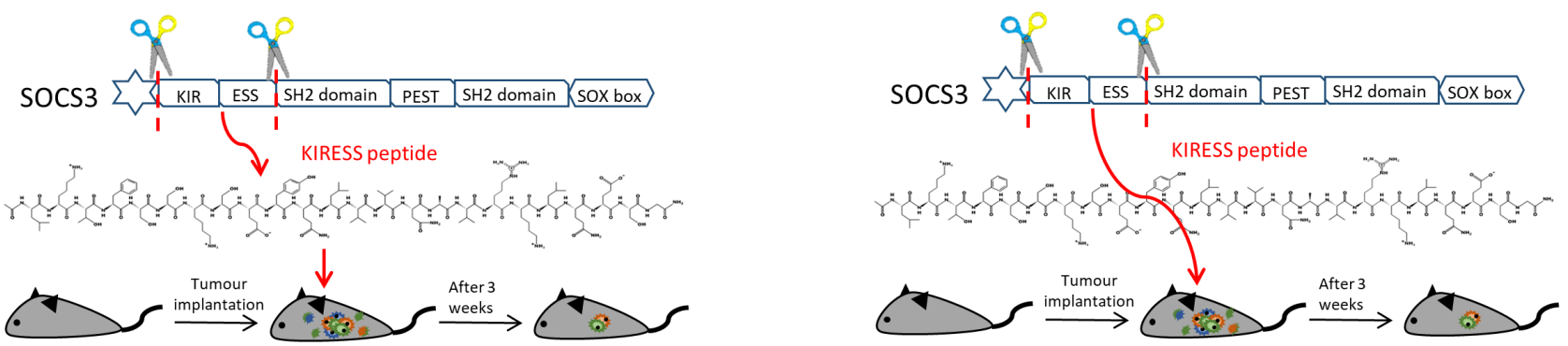

Figure 1: Schematic representation of hypothetical mechanism of action of SOCS3 peptidomimetics based on KIRESS domain.

The activity of KIRESS peptide was evaluated in vivo in mouse xenografts model of human TNBC subtype, MDA-MB-231-luci tumour. Interestingly, mice treated with KIRESS peptide showed a marked decrease in tumour growth. Moreover, it revealed able to completely eliminate pulmonary metastasis. At molecular level, STAT3 and NF-kB were identified as key intracellular targets of KIRESS peptide, since their phosphorylation was strongly reduced by its administration confirming that KIRESS is able to mimic the action of the entire SOCS3 protein. Fortunately, in histopathological examination of treated animals there was no observable toxicity caused by the peptide.

These overall data clearly indicate that KIRESS peptide can be considered a valid starting point to build, through structural and chemical modifications, compounds with high affinity and stability as potential therapeutics in TNBC. 


\section{References}

[1] Carow B, Rottenberg ME. SOCS3, a Major Regulator of Infection and Inflammation. Frontiers in immunology 2014;5: 58.

[2] Yoshimura A, Yasukawa H. JAK's SOCS: a mechanism of inhibition. Immunity 2012;36: 157-9.

[3] Ahmed, C. M.; Dabelic, R.; Bedoya, S. K.; Larkin, J., 3rd; Johnson, H. M., A SOCS1/3 Antagonist Peptide Protects Mice Against Lethal Infection with Influenza A Virus. Front Immunol2015, 6, 574.

[4] Balabanov, R.; Strand, K.; Goswami, R.; McMahon, E.; Begolka, W.; Miller, S. D.; Popko, B., Interferongamma-oligodendrogte interactions in the regulation of experimental autoimmune encephalomyelitis. J

Neurosci 2007, 27 (8), 2013-24.

[5] Kim G, Ouzounova M, Quraishi AA, Davis A, Tawakkol N, Clouthier SG, Malik F, Paulson AK, D’Angelo RC, Korkaya S, Baker TL, Esen ES, et al. SOCS3-mediated regulation of inflammatorycytokines in PTEN and p53 inactivated triple negative breast cancer model. Oncogene 2015;34: 671-80.

[6] Doti, N., Scognamiglio, P. L., Madonna, S., Scarponi, C., Ruvo, M., Perretta, G., Albanesi, C., and Marasco, D. (2012) New mimetic peptides of the kinase-inhibitory region (KIR) of SOCS1 through focused peptide libraries, The Biochemical journal 443, 231-240.

[7] La Manna, S.; Lopez-Sanz, L.; Leone, M.; Brandi, P.; Scognamiglio, P. L.; Morelli, G.; Novellino, E.; Gomez-Guerrero, C.; Marasco, D., Structure-activity studies of peptidomimetics based on kinase-inhibitory region of suppressors of cytokine signaling 1. Biopolymers 2017; e23082.

[8] Kershaw NJ, Murphy JM, Liau NP, Varghese LN, Laktyushin A, Whitlock EL, Lucet IS, Nicola NA, Babon JJ. SOCS3 binds specific receptor-JAK complexes to control cytokine signaling by direct kinase inhibition. Nature structural \& molecular biology 2013;20: 469-76.

[9] La MannaS, Lee E, Ouzounova M, Di Natale C, Novellino E, Merlino A, Korkaya H, Marasco D. Mimetics of Suppressor of cytokine signalling 3: novel potential therapeutics in triple breast cancer. International Journal of Cancer 2018;143: 2177-2186. 